\title{
化学发光法测定超氧化物歧化酶的 动力学研究
}

\author{
方允中 刘智峰 李益新 黄素敏 \\ (军事医学科学院, 北京)
}

超氧化物自由基 $\left(\mathrm{O}_{2}^{-}\right)$可自动歧化为 $\mathrm{O}_{2}$ 和 $\mathrm{H}_{2} \mathrm{O}_{2}$, 但在超氧化物歧化酶 (SOD) 的催化下, 其反应速度可增加 4 5 个数量级 ${ }^{[1]}$. 由于 $O_{i}^{-}$很不稳定, 在 SOD 的动力学研究中, 常采用脉 冲射解等直接法 ${ }^{[2]}$, 但后者需要昂贵的仪器设备, 故不适合于一般应用. 很多间接法尚不能取 代直接法, 应用于动力学研究. 化学发光法灵敏度虽高, 专一性虽强, 但和很多间接法一样, 也 必须根据抑制反应的百分数和 SOD 浓度的关系曲线, 确定抑制 $50 \%$ 反应的相应浓度, 才能计 算 $S O D^{[3]}$. 这种根据曲线, 而不是根据直线的计算方法, 不仅在使用上很不方便, 而且也不适 合动力学研究要求. 为了解决这个问题, 我们推导了化学发光法测定 SOD 的动力学公式, 并 得到实验证据。

\section{材 料 和 方 法}

SOD 采用 McCord-Fridovich 方法 ${ }^{[4]}$ 从牛红细胞提纯. 每 $\mathrm{mg}$ 的酶制品为 3000 以上. 的 McCord-Fridovich 单位.

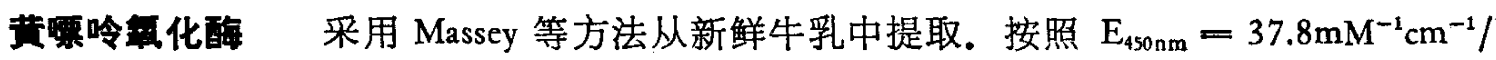
结合酶的 FAD 分子,计算酶的浓度.

细胞色秦 C (III 型) Sigma 公司产品.

黄霓呤 $\mathrm{BDH}$ 公司产品.

\section{3-基邻苯二甲酰胼 (luminol) Aldrich 公司产品.}

细胞色率 $\mathrm{c}$ 还原法测 $\boldsymbol{k}_{\mathrm{SOD}}$ 采用 Sawada 与 Yamazaki 法 ${ }^{[6]}$ ，但反应体系为 $\mathrm{pH} 10.2$ 的 $0.05 M$ 碳酸盐缓冲液配成的 $1.7 \times 10^{-5} M$ 细胞色素 $c 、 0.1 \mathrm{~m} M$ 黄嘌呤和 $10.2 \mathrm{n} M$ 黄嘌呤氧 化酶. 细胞色素 $\mathrm{c}$ 还原量系按照 $\Delta E_{550 \mathrm{~nm}}=19.6 \mathrm{~m} \mathrm{M}^{-1} \mathrm{~cm}^{-1}$ 计算. 在 $25^{\circ} \mathrm{C}$, 使用日立 557 型 分光光度计测定光吸收.

化学发光法测定 $\boldsymbol{k}_{\boldsymbol{L}}$ 其手续与测定 $\mathrm{SOD}^{[3]}$ 一致,但反应体系与细胞色素 $\mathrm{c}$ 还原法测 定 $k_{\mathrm{sOD}}$ 基本相同,所不同的是用 $1 \times 10^{-4} M$ luminol 代替细胞色素 c. 在 $25^{\circ} \mathrm{C}$, 使用 $\mathrm{LKB}$ Wallac 1250 型发光仪测量化学发光强度.

\section{结果与 讨 论}

1. 化学发光测定 SOD 的动力学公式推导 在此法中产生 $O_{i}^{-}$的体系为黄嘌呤-黄嘌 呤氧化酶. $\mathrm{O}_{i}^{-}$的产量虽依赖 $\mathrm{pH} 、 \mathrm{O}_{2}$ 与黄嘌呤的浓度，但在规定的实验条件下， $\mathrm{O}_{i}^{-}$处于稳

本文 1985 年 3 月 8 日收到. 
定态.

$\mathrm{O}_{i}^{\overline{-}}$ 与 luminol 反应, 产生激发态产物. 后者转变为基态产物, 同时放出光. 设反应速度 为 $V_{L}$, 反应速度常数为 $k_{L}$, 则 $V_{L}=k_{L}\left[\mathrm{O}_{2}^{-}\right]$[luminol].

又设 $\mathrm{SOD}$ 催化下 $\mathrm{O}_{i}^{-}$的歧化反应速度为 $V_{S O D}$, 反应速度常数为 $k_{S O D}$, 则 $V_{S O D}=$ $k_{S O D}\left[O_{i}^{\bar{i}}\right][S O D]$. $O_{i}^{\bar{z}}$ 反应的总速度 $(V)=V_{L}+V_{S O D}$.

$$
\frac{V}{V_{L}}=1+\frac{V_{\mathrm{SOD}}}{V_{L}}=1+\frac{k_{\mathrm{SOD}}\left[\mathrm{O}_{\dot{2}}^{-}\right][\mathrm{SOD}]}{k_{L}\left[\mathrm{O}_{\bar{i}}^{-}\right][\text {luminol }]}=1+\frac{k_{\mathrm{SOD}}}{k_{L}[\text { luminol }]} \cdot[\mathrm{SOD}] \text {. }
$$

在测定 SOD 的过程中, luminol 浓度变动很小, 故 $\frac{k_{\mathrm{SOD}}}{k_{L} \text { [luminol] }}$ 可看成为另一常数 $k$.上 式遂可简化为 $\frac{V}{V_{L}}=1+k[S O D]$.

2. $\boldsymbol{k}_{\text {SOD }}$ 和 $\boldsymbol{k}_{\boldsymbol{L}}$ 值的测定虽然 $k_{S O D}$ 值可用细胞色素 $\mathrm{c}$ 还原法测定, 但用化学发光 法测定 $k_{L}$ 时, 必须采用细胞色素还原法中同样反应体系. 我们选择了 $\mathrm{pH} 10.2$ 体系测定 $k_{\text {SOD }}$ 和 $k_{L}$.

根据细胞色素 $\mathrm{c}$ 还原动力学, 测定 $k_{\mathrm{sod}}$ 时, 应首先测定 $k_{\mathrm{c}} . \mathrm{O}_{\dot{i}}^{-}$还原细胞色素 $\mathrm{c}$ (氧化 型 $)$ 的反应速度 $\left(V_{\mathrm{c}}\right)=k_{\mathrm{c}}\left[\mathrm{Cyt} \mathrm{c}\left(\mathrm{Fe}^{3+}\right)\right]\left[\mathrm{O}_{\dot{i}}^{-}\right]$. 同时 $\mathrm{O}_{\dot{i}}^{-}$还可自动歧化, 其反应速度 $\left(V_{\mathrm{d}}\right)=$ $k_{\mathrm{d}}\left[\mathrm{O}_{i}^{-}\right]$.

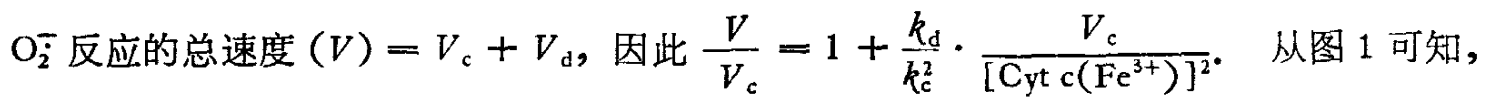
直线斜度 $\left(\frac{k_{\mathrm{d}}}{k_{\mathrm{c}}^{2}}\right)=1.81 \times 10^{-3} \mathrm{M} \cdot \mathrm{sec}$. 据 Rabani 与 Nielsen ${ }^{[7]}$ 报道,用脉冲射解法得出 $\mathrm{pH}$ 10.2 体系中 $k_{\mathrm{d}}$ 为 $3.1 \times 10^{2} M^{-1} \cdot \mathrm{sec}^{-1}$, 代人上式, 则可计算出 $k_{\mathrm{c}}=4.14 \times 10^{2} \mathrm{M}^{-1} \mathrm{sec}^{-1}$. 重 复测定得到一致结果.

在 SOD 存在下, $\frac{V}{V_{\mathrm{c}}}=\frac{k_{\mathrm{SOD}}}{k_{\mathrm{c}}\left[\mathrm{C}_{\mathrm{yt}} \mathrm{c}\left(\mathrm{Fe}^{+3}\right)\right]} \cdot[\mathrm{SOD}]\left(V=V_{\mathrm{c}}+V_{\mathrm{SOD}}, V_{\mathrm{SOD}}=k_{\mathrm{SOD}}[\mathrm{SOD}]\right.$ $\left[\mathrm{O}_{i}^{-}\right]$). 依照图 2 所示, $\frac{V}{V_{c}}$ 与 [SOD] 确成直线相关, 其斜率 $\left(\frac{k_{\mathrm{SOD}}}{k_{\mathrm{c}}\left[\mathrm{Cyt} \mathrm{c}_{\mathrm{yt}}\left(\mathrm{Fe}^{3+}\right)\right]}\right)$ 为 $1.5 \times$ $10^{-5}$. 从而计算出 $k_{S O D}$ 为 $1.06 \times 10^{8} M^{-1} \cdot \mathrm{sec}^{-1}$. 重复测定结果是一致的.

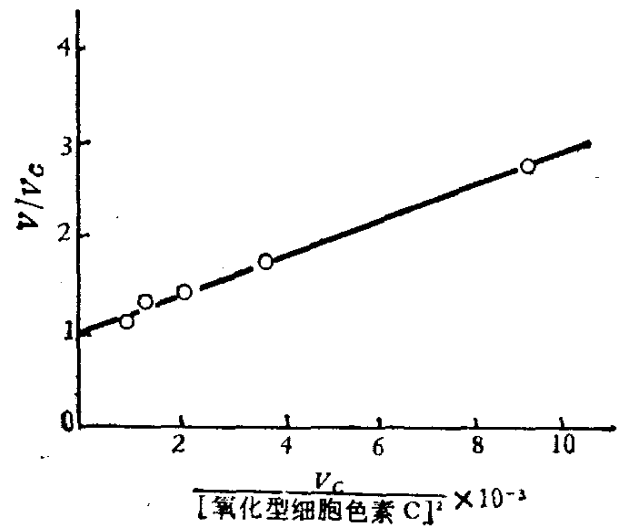

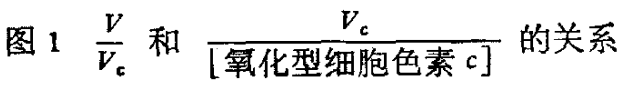

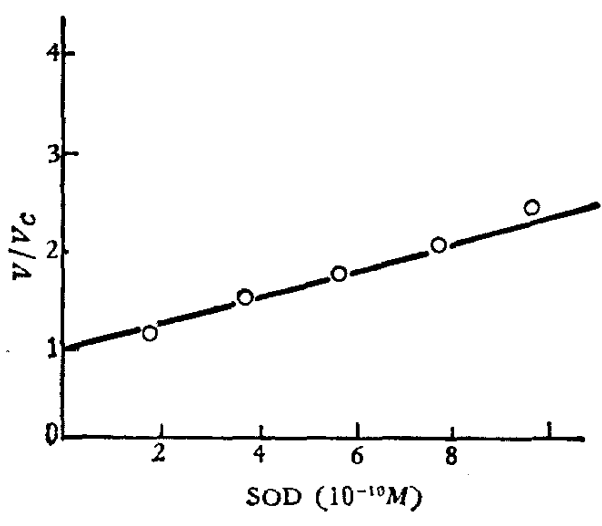

图 $2 \frac{V}{V_{c}}$ 和 SOD 浓度的关系 
采用上述的测定 $k_{\mathrm{SOD}}$ 的体系, 但将细胞色素 $\mathrm{c}$ 改为 $1 \times 10^{-4} M$ luminol, 得出 $\mathrm{O}_{\dot{2}}^{-}$和 luminol 反应的产物放出化学发光的条件下，不同浓度的 SOD 对发光抑制的百分数. 按照

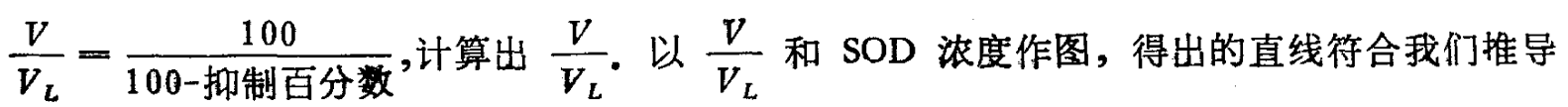
的动力学公式(图 3). 其斜率 $\left(\frac{k_{\mathrm{soD}}}{k_{L}[\text { luminol }]}\right)=0.86 \times 10^{10} \mathrm{M} \cdot \mathrm{sec}$. 由于 [luminol] 为 $1 \times$ $10^{-4} \mathrm{M}, k_{\mathrm{soD}}$ 为 $1.06 \times 10^{8} \mathrm{M}^{-1} \mathrm{sec}^{-1}$, 故 $k_{L}=1.23 \times 10^{2} \mathrm{M}^{-1} \mathrm{sec}^{-1}$.

3. 动力学公式在化学发光法中的应用虽然化学发光法测定 SOD 时, 发光反应抑制 的百分数与 SOD 浓度的关系图形是曲线的,但是按照我们推导的动力学公式计算和 $\frac{V}{V_{L}}$ 和 SOD 浓度作图,却成直线相关. 该动力学公式不仅符合纯酶溶液中 SOD 的测定, 而且也符合 人的红细胞溶血液、大閶组织匀浆以及黎豆粉浸出液等许多生物样品中 SOD 测定结果(图 4 和图5). 这表明该公式不仅有理论依据,而且有实验证据. 它在化学发光法中的应用使 SOD 的计算更为方便,而且测定结果也更为准确.

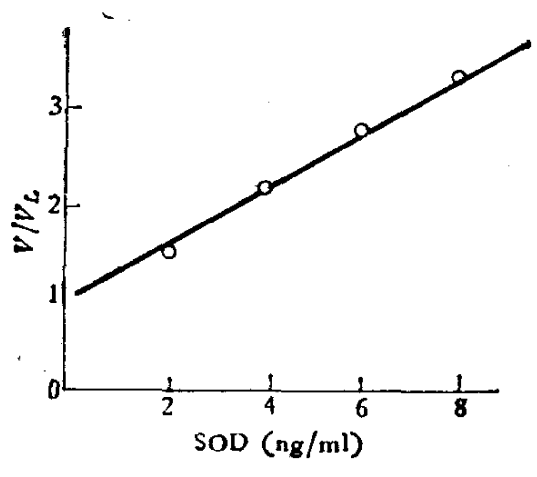

图 $3 V / V_{L}$ 和 SOD 浓度的关系

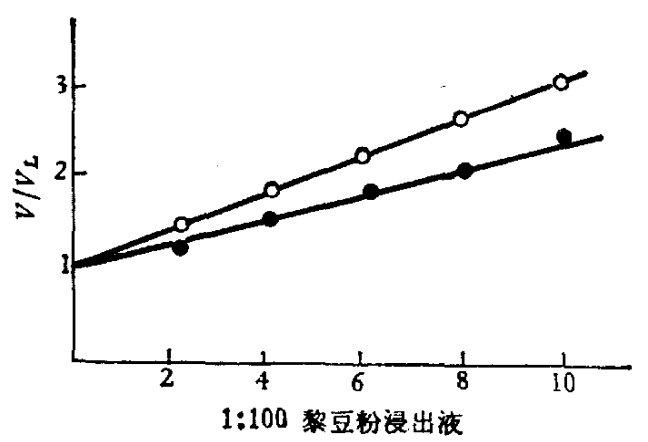

图 $5 V / V_{L}$ 和不同量的黎豆粉浸出液的直线关系 1 号样品 - $-\operatorname{SOD} 47 \mu \mathrm{g} / \mathrm{ml}$; 2 号样品 $0-0$ SOD $70 \mu \mathrm{g} / \mathrm{ml}$
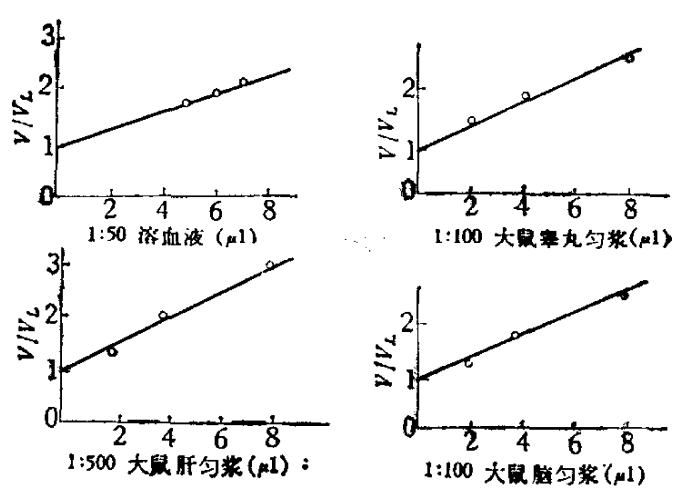

图 $4 V / V_{L}$ 和溶直菠或大鼠组织匀浆用量之间的关系

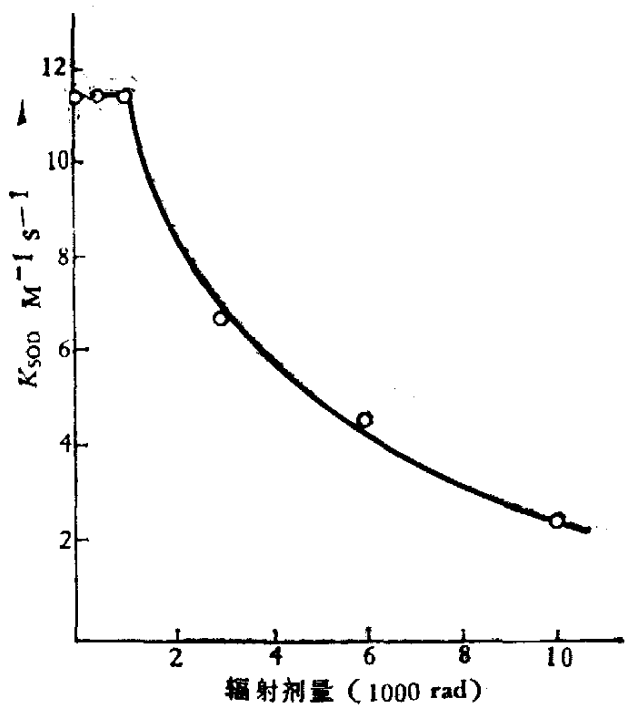

图 6 电离辐射对 $K_{\mathrm{SOD}}$ 的影响 $0.01 \mathrm{mg} / \mathrm{ml}$ SOD (系用 $\mathrm{pH} 7.8,0.05 \mathrm{M}$ 磷酸缓冲液配制)照射不同剂量 
4. 动力学公式在电离辐射对 $\boldsymbol{k}_{\mathrm{SOD}}$ 的影响研究中的应用 取 $0.01 \mathrm{mg} / \mathrm{ml}$ 的 SOD 溶液 (用 $\mathrm{pH} 7.8 、 0.05 M$ 磷酸缓冲液配制)，照射 400、1000、3000、6000 或 $10000 \mathrm{rad}$. 依照图 6 所

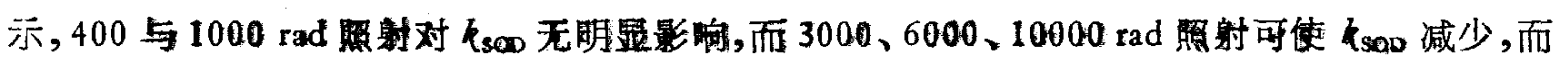
且减少程度随着照射剂量而加剧. 这表明, 照射后 SOD 的理化性质可能发生改变. 这个 结果与前次报告 ${ }^{[8]}$ 中 $10000-100000 \mathrm{rad}$ 照射使 SOD 活性减低和其理化性质改变的结果是一 致的.

\section{参考文城}

[ 1 ] MeCord, J. M., Crips, J. D., Fridovich, I., Superaxide and Superoxide Dismutase (Eds. J. M. Micheison, J. M. McCord, I. Fridovich), Academic Press, New York, 1977, 11-17.

[2] Klug, D., Rabani, J., Fridovich, I., J. Biol. Chem., 247(1972), 4839-4842.

[ 3 ] 柰益新、方允中，生物化学与生物物理进展，2(1983)，59-63.

[ 4 ] McCord, J. M., Fridovich, I., J. Bial. Chem., 244 (1969), 6049-6055.

[ 5 ] Massey, V., Brunby, P. E., Komai, H., J. Biol. Chem, 244(1969), 1682-1961.

[ 6 ] Sawada, Y., Yamazaki, I., Biochim. Biophys. Acta, 327(1973), 257-265.

[ 7 ] Rabani, J., Nielsen, S. C., J. Phys. Chem.x 73(1969), 3736-3744.

[8] 方允中、刘智萧、本益新, 科学通腿, $29(1984), 15: 943-946$ 。 